\title{
DRYMONIA TOMENTULIFERA, SP. NOVA DE COSTA RICA, Y NOTAS SOBRE LA BIOLOGÍA REPRODUCTIVA DEL GÉNERO DRYMONIA (GESNERIACEAE: EPISCIEAE)
}

\author{
RICARDO KRIEBEL \\ Department of Biology, San Francisco State University, 1600 Holloway Av., San Francisco, CA 94132, USA. \\ Instituto Nacional de Biodiversidad, apdo. 22-3100, Santo Domingo, Heredia, Costa Rica \\ rkriebel@calacademy.org
}

\begin{abstract}
A new species of the neotropical genus Drymonia, D. tomentulifera, is described and illustrated, and compared to D. pilifera. Notes on the reproductive biology, especially the widespread occurrence of protandry in the genus Drymonia are included.
\end{abstract}

Resumen. Se describe e ilustra una nueva especie del género neotropical Drymonia, D. tomentulifera, y se compara con D. pilifera. Se incluyen notas sobre la biología reproductiva del género Drymonia, con énfasis en la protandria.

Palabras claves / Key words: Gesneriaceae, Drymonia pilifera, Drymonia tomentulifera, polinización / pollination, dicogamia / dichogamy, protandria / protandry, Costa Rica

Drymonia Mart. es el segundo género más diverso de la familia Gesneriaceae en Costa Rica, donde se conocen 23 especies. A la vez, en este país es el más diverso en formas de vida, morfología y color floral y tipos de frutos; es el único que incluye cápsulas carnosas bivalvadas, con endocarpo dehiscente o indehiscente, y bayas (Clark et al. 2006, Kriebel 2006). El género más diverso, Columnea, es polinizado aparentemente sólo por colibríes, mientras que varias especies de Drymonia son polinizadas por abejas (Apidae: Euglossini y Anthophorini). El color principalmente blanco de la corola en las especies polinizadas por abejas indica que varias especies costarricenses de Drymonia posiblemente son polinizadas por éstas; solamente en $D$. conchocalyx, $D$. coriacea y $D$. rubra se han observado colibríes como posibles polinizadores (obs. pers.) y dos de las tres presentan el síndrome ornitofílico (D. coriacea y D. rubra). En otras especies con corolas moradas, como D. glandulosa, o amarillas como en $D$. parviflora, los colibríes también podrían ser importantes polinizadores. No obstante, hasta ahora se carece de observaciones que lo confirmen.

El trabajo más completo sobre polinización en Drymonia fue realizado por Kim Steiner (1985). Este autor comprobó que machos y hembras de dos especies de abejas del género Epicharis (E. monozona y E. rustica) buscan néctar y polinizan $D$. serrulata en Panamá. Además, Steiner señaló la importancia de los tricomas glandulares en la parte superior del tubo de la corola, que facilitan la adherencia del polen al tórax de las abejas. Dressler (1968) observó la polinización de $D$. turrialvae por machos de Eulaema nigrifacies; Wieher (1977) comunica que machos y hembras de Euglossa gibbosa visitan las flores de Drymonia ecuadorensis en Ecuador. Observaciones adicionales sobre polinización de otras especies de Drymonia se halan en Steiner (1985).

En su detallado estudio de Drymonia serrulata, Steiner (1985) describió la separación temporal del sexo (dicogamia), en las flores de dicha especie, por el fenómeno de protandria (fase masculina precede a la fase femenina). La protandria ha sido asociada con polinización biótica por insectos y otros animales (Lloyd \& Webb 1986), como es el caso en Drymonia; la protoginia, por otro lado, ha sido asociada con polinización abiótica, principalmente por viento (Bertin \& Newman 1993, Sargent \& Otto 2004). Para que la protandria funcione, debe haber una recompensa para el polinizador en ambas fases sexuales; en el caso de Drymonia, esta recompensa es el néctar. Todas las especies producen néctar, la mayoría por una o dos glándulas localizadas en el dorso del ovario. El néctar se acumula en el espolón basal de la corola y se compone principalmente de sacarosa (Steiner 1985). Los dos trabajos más completos sobre dicogamia que pude encontrar incluyen solamente ocho especies de Gesneriaceae (Bertin \& Newman 1993) o ninguna (Sargent \& Otto 2004). Aunque se ha hipotetizado que el beneficio de la dicogamia es evitar la autopolinización, Bertin \& Newman (1993) señalan ocho explicaciones 
alternativas, de varios autores, para explicar el posible beneficio de este mecanismo.

En este artículo se describe una nueva especie de Drymonia, D. tomentulifera, endémica en Costa Rica, que es protándrica. De las 23 especies de este género que habitan en Costa Rica, 21 son protándricas. Por la homogeneidad de las glándulas nectaríferas y la presencia de un espolón basal en la corola, donde se acumula el néctar, es posible que todas las $c a .140$ especies del género sean protándricas.

Otros géneros gesneriáceos de Costa Rica, en los que he observado protandria, son Alloplectus Mart., Besleria L., Columnea L., Glossoloma Hanst., Koellikeria Regel, Kohleria Regel, Paradrymonia Hanst., Rhynchoglossum Blume y Rufodorsia Wiehler.

\section{Drymonia tomentulifera Kriebel, sp. nova}

A Drymonia pilifera Wiehler affinis, sed indumento tomentuloso, corola minore, maculata, cum superficie externa sericea differt.

Tipo: Costa Rica: Alajuela; cuenca del Sarapiquí, Virgen del Socorro, $10^{\circ} 15^{\prime} 25^{\prime}$ 'N, 84 $4^{\circ} 10^{\prime} 20^{\prime \prime} \mathrm{W}, 800 \mathrm{~m}$, 21 jul 2002, R. Kriebel 536 (Holotipo: INB, isotipos: $\mathrm{CR}, \mathrm{MO})$. Fig. 1

Enredadera del sotobosque en bosque poco alterado, con tallos tomentulosos especialmente hacia el ápice, más o menos teretes, verdes; entrenudos $0.6-9 \mathrm{~cm}$ de largo y 3-5 mm de diámetro. Hojas de cada nudo de igual tamaño a menos frecuentemente desiguales, pecioladas; pecíolos $0.5-2.5 \mathrm{~cm}$ de largo y $1-2 \mathrm{~mm}$ de diámetro, tomentulosos, verdes; láminas 2-10.5 x 1.3-6 cm, elípticas a elíptico-ovadas, escabrosas en el haz, tomentulosas en las venas principales del envés y escabrosas entre éstas, cartáceas, el ápice agudo, la base aguda a obtusa y en ocasiones asimétrica, el margen crenado a aserrado, venas secundarias 7-9 pares, prominentes en el envés. Inflorescencia de flores axilares solitarias; pedúnculos ausentes; brácteas filiformes, 5-7 mm de largo; pedicelos $6-1.6 \mathrm{~cm}$ de largo, tomentulosos. Lóbulos del cáliz 5, verdes, ovados, 2.5-3.1 cm de largo y 1.5-2 cm de ancho, libres casi hasta la base y connados por $c a .2 \mathrm{~mm}$, la base cordada, el ápice agudo, el margen serrulado a serrado; corola 3.2-3.6 cm, el tubo externamente blanco o crema, internamente con líneas moradas y los lóbulos con manchas moradas; externamente glabra en la base y serícea a menos frecuentemente seríceo-vilosa en el tubo y hacia la boca, internamente con tricomas glandulares hacia la boca, el espolón $3 \mathrm{~mm}$ de largo, el tubo levemente arqueado, la boca bilabiada, el margen de los lóbulos eroso, los tres ventrales de mayor tamaño, especialmente el central, los cinco 0.4-0.6 x 0.5-0.7 cm; filamentos ca. 2.5-3 cm de largo, glabros, connados entre sí y adnados a la base de la corola por 0.4-0.9 mm; anteras de 2-2.2 x $1 \mathrm{~mm}$; disco reducido a una glándula nectarífera dorsal, glabra y de ápice agudo; ovario 5-6 $\mathrm{mm}$ de largo, seríceo; estilo ca. 2.7-3.2 cm de largo, glabro, estigma fuertemente bilobado y papiloso. Fruto morado, dehiscente, con dos valvas también moradas internamente.

Distribución, HÁBitat y Biología. Drymonia tomentulifera es hasta ahora endémica en Costa Rica, donde crece en bosques muy húmedos de la vertiente caribe de la Cordillera de Tilarán y de la Central, entre 400 y $1000 \mathrm{~m}$ de altitud. En el Parque Nacional Braulio Carrillo, entre 400 y $500 \mathrm{~m}$ de altitud, D. tomentulifera crece simpátricamente con otras especies del género, como $D$. submarginalis, $D$. turrialvae y $D$. warszewicziana. Otros géneros de la familia simpátricos con Drymonia en esta localidad son Besleria, Columnea, Napeanthus, Neomortonia, Paradrymonia y Rufodorsia.

Drymonia tomentulifera es protándrica, fenómeno reproductivo fácil de verificar en las Gesneriáceas, ya que en la fase masculina los filamentos se encuentran rectos, los estambres están ubicados en la parte superior de la boca de la corola y el estigma se encuentra en crecimiento. En el caso de $D$. tomentulifera, como el estigma es bilobado se sabe que todavía está en desarrollo porque los lóbulos no se han separado para exponer la parte receptiva. Este aspecto de la madurez del estigma también fue observado y descrito en detalle por Steiner (1985) en D. serrulata. En la fase femenina los filamentos ya se han retraído, los estambres se encuentran sin polen, el estilo se ha desarrollado en su totalidad y los dos lóbulos del estigma ya se han desenvuelto (Fig. 1A). A continuación se presenta un cuadro de las especies de Drymonia de Costa Rica, de las cuales 21 han sido confirmadas como protándricas por observaciones de campo y estudio de especímenes. 
Cuadro 1. Especies de Drymonia que habitan en Costa Rica, cada una con un testigo (INB), en el que se verificó la protandria. Los signos de pregunta corresponden a especies con un número insuficiente de especímenes con flores para verificar la protandria. El asterisco corresponde a especímenes con flores preservadas en alcohol.

Especie

Drymonia alloplectoides Hanst.

Drymonia conchocalyx Hanst.

Drymonia coriacea (Oerst. ex Hanst.) Wiehler

Drymonia fimbriata C.V. Morton

Drymonia folsomii L.E. Skog

Drymonia glandulosa Kriebel

Drymonia lanceolata (Hanst.) C.V. Morton

Drymonia macrantha (Donn. Sm.) D.N. Gibson

Drymonia macrophylla (Oerst.) H.E. Moore

Drymonia mortoniana Wiehler

Drymonia multiflora (Oerst. ex Hanst.) Wiehler

Drymonia parviflora Hanst.

Drymonia peltata (Oliv.) H.E. Moore

Drymonia pilifera Wiehler

Drymonia rubra C.V. Morton

Drymonia rubripilosa Kriebel

Drymonia serrulata (Jacq.) Mart.

Drymonia stenophylla (Donn. Sm.) H.E. Moore

Drymonia submarginalis Gómez-Laur. \& Chavarría

Drymonia tomentulifera Kriebel

Drymonia turrialvae Hanst.

Drymonia uninerva Wiehler

Drymonia warszewicziana Hanst.
Testigo

R. Kriebel 2471

A. Martínez 120

R. Kriebel \& D. Solano 2473 *

M. Grayum et al. 10600

C. Cano 103

R. Kriebel 4414

R. Kriebel 1912*

M.M. Chavarría 760 *

E. Rojas 144

?

R. Kriebel 316 *

E. Alfaro $264 *$

B. Hammel 21844 *

C. Moraga $651 *$

R. Kriebel s.n. *

R. Kriebel s.n. *

K.E. Steiner (1985)

G. Herrera 2973

R. Kriebel et al. 3991

R. Kriebel 5111*

A. Rodríguez \& L. Vargas 2700

?

R. Kriebel \& J. Larraguivel 649
Afinidades taxonómicas. Drymonia tomentulifera es muy similar a $D$. pilifera Wiehler de Nicaragua, Costa Rica y Panamá, con la cual comparte el hábito de enredadera, pedicelos cortos, lóbulos del cáliz ovados, con el margen serrulado a aserrado, corola blanca a crema por fuera, con el tubo levemente curvo en la base pero recto hacia la boca, y estigma bilobado. Es muy posible que ambas especies crezcan simpátricamente, ya que su distribución geográfica y altitudinal es muy similar. Sin embargo, la distribución de $D$. pilifera es más amplia y se extiende desde el Río San Juan, en Nicaragua, hacia el sur hasta la Cordillera de Talamanca y las provincias panameñas de Bocas del Toro, Chiriquí, Coclé, San Blas y Veraguas. Ambas especies se pueden distinguir con la dicotomía siguiente:

Indumento de ramitas, hojas, pedicelos y cáliz piloso; cáliz secando verde-hialino; corola $4.5 \mathrm{~cm}$ de largo, con los lóbulos de 0.7-0.9 x 0.8-1.1 cm, rojizos a morados en su totalidad, el tubo viloso externamente D. pilifera

Indumento de ramitas, hojas, pedicelos y cáliz tomentuloso; cáliz secando verde opaco; corola 3.2-3.6 $\mathrm{cm}$ de largo, con los lóbulos de 0.4-0.6 x 0.5-0.7 cm, manchados de morado pero no pintados en su totalidad, el tubo seríceo externamente ......... D. tomentulifera

Para obtener medidas adecuadas de las flores, se preservaron en INB corolas de Drymonia tomentulifera (R. Kriebel 5111) y D. pilifera (C. Moraga 651) en alcohol diluido al $70 \%$.

Etimología. El epíteto hace referencia al indumento tomentuloso de ramitas apicales, hojas, pedicelos y 
cáliz de la nueva especie.

Paratipos. Costa Rica: Alajuela; Poás, Laguna Echandi, entre San Miguel, Vara Blanca, $10^{\circ} 04^{\prime} 00^{\prime \prime} \mathrm{N}$, $84^{\circ} 14^{\prime} 45^{\prime \prime O}, 1000$ m (fr), 3 mar 1991, M.M. Chavarría 452 (INB); loc. cit., Upala, Llanura de Guatuso, San Rafael de Guatuso, 1042'43”N, 84⒌'58'O, 400$500 \mathrm{~m}$ (fl), 15 sept 1988, M.M. Chavarría 320 (INB). Guanacaste; Tilarán, 1-2 km west of Lago de Cote, 13 $\mathrm{km}$ north of Tilarán, continental divide on SE slope of Volcán Tenorio, 10³5'00'N, 8456'00'O, 800-900 m, 24 ago 1993 (fl), W. Haber \& W. Zuchowski 11658 (CR, INB, MO). Heredia; Sarapiquí, Rara Avis, ca. 15 km al suroeste de Horquetas, camino Catarata y Camino Real por el puente "Sanguijuelero", $10^{\circ} 17^{\prime} \mathrm{N}, 84^{\circ} 02^{\prime} \mathrm{O}$, 400-600 m, 7 nov 1989 (fl), O. Vargas 285 (INB). Limón; Pococí, Parque Nacional Braulio Carrillo, Quebrada González, Sendero Las Palmas, $10^{\circ} 09^{\prime} 15^{\prime} \mathrm{N}$, 8356'30'O, 500-600 m, 13 dic 2002 (fl), R. Kriebel \& $J$. Larraguivel 1853 (INB); loc. cit., 26 dic 2005 (fl), $R$. Kriebel 5111 (INB).

Agradecimientos. A Reinaldo Aguilar y al proyecto Flórula Digital de la Estación Biológica La Selva por la imagen de Drymonia pilifera; a Calixto Moraga por la corola en líquido de D. pilifera; a Fanny Montero, Frank González y Daniel Santamaría por su apoyo en el procesamiento y la localización de especímenes; a John L. Clark y a Carlos O. Morales por los comentarios sobre el manuscrito.

LITERATURA CITADA

Bertin, R.I. \& C.M. Newman. 1993. Dichogamy in Angiosperms. Bot. Rev. 59: 153-154.

Clark, J.L., P.S. Herendeen, L.E. Skog \& E.A. Zimmer. 2006. Phylogenetic relationships and generic boundaries in the Episcieae (Gesneriaceae) inferred from nuclear, chloroplast, and morphological data. Taxon 55(2): 313-336.

Dressler, R.L. 1968. Observations on orchids and euglossine bees in Panama and Costa Rica. Rev. Biol. Trop. 15: 143-183.

Lloyd, D.G. \& C.J. Webb. 1986. The avoidance of interference between the presentation of pollen and stigmas in angiosperms. 1. Dichogamy. New Zealand J. Bot. 24: 135-162.

Kriebel, R. 2006. Anew species and notes on Drymonia (Gesneriaceae) from Costa Rica. Novon 16: 65-68.

Sargent, R.D. \& S.P. Otto. 2004. A phylogenetic analysis of pollination mode and the evolution of dichogamy in angiosperms. Evol. Ecol. Res. 6: 1183-1199.

Steiner, K.E. 1985. The Role of Nectar and Oil in the Pollination of Drymonia serrulata (Gesneriaceae) by Epicharis Bees (Anthophoridae) in Panama. Biotropica 17(3): 217-229.

Wiehler,H. 1977. New genera and species of Gesneriaceae from the Neotropics. Selbyana 2: 67-132. 

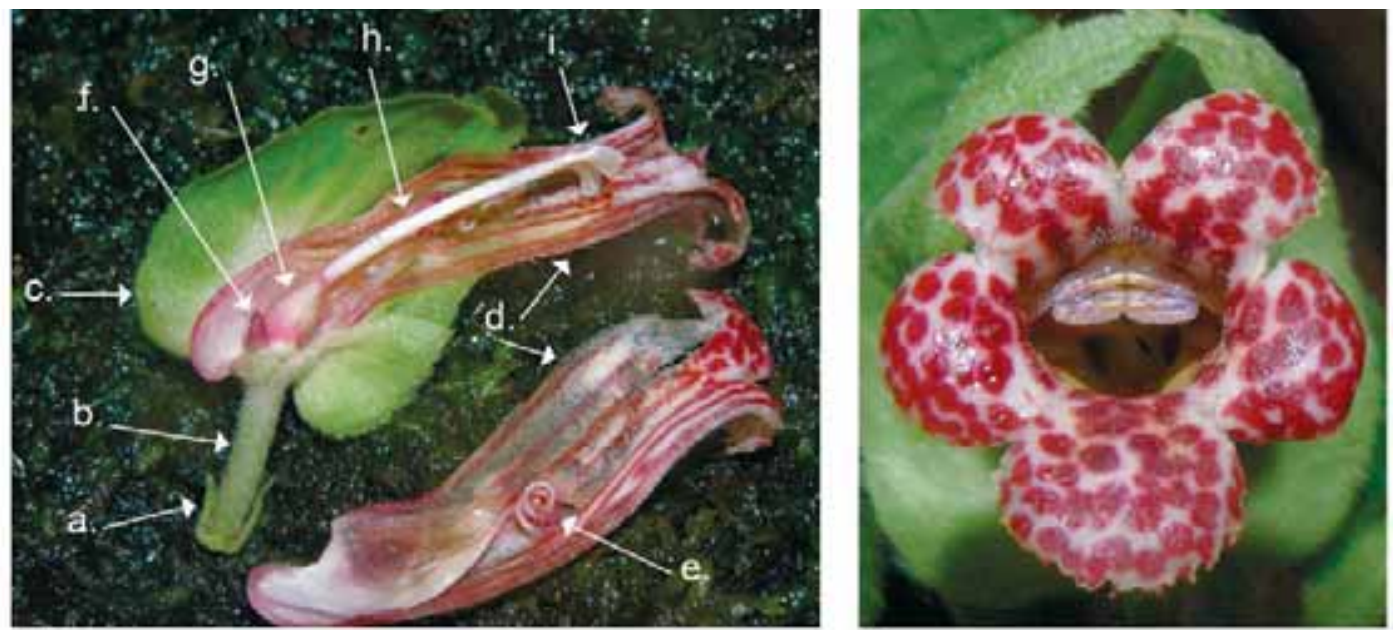

\section{A.}
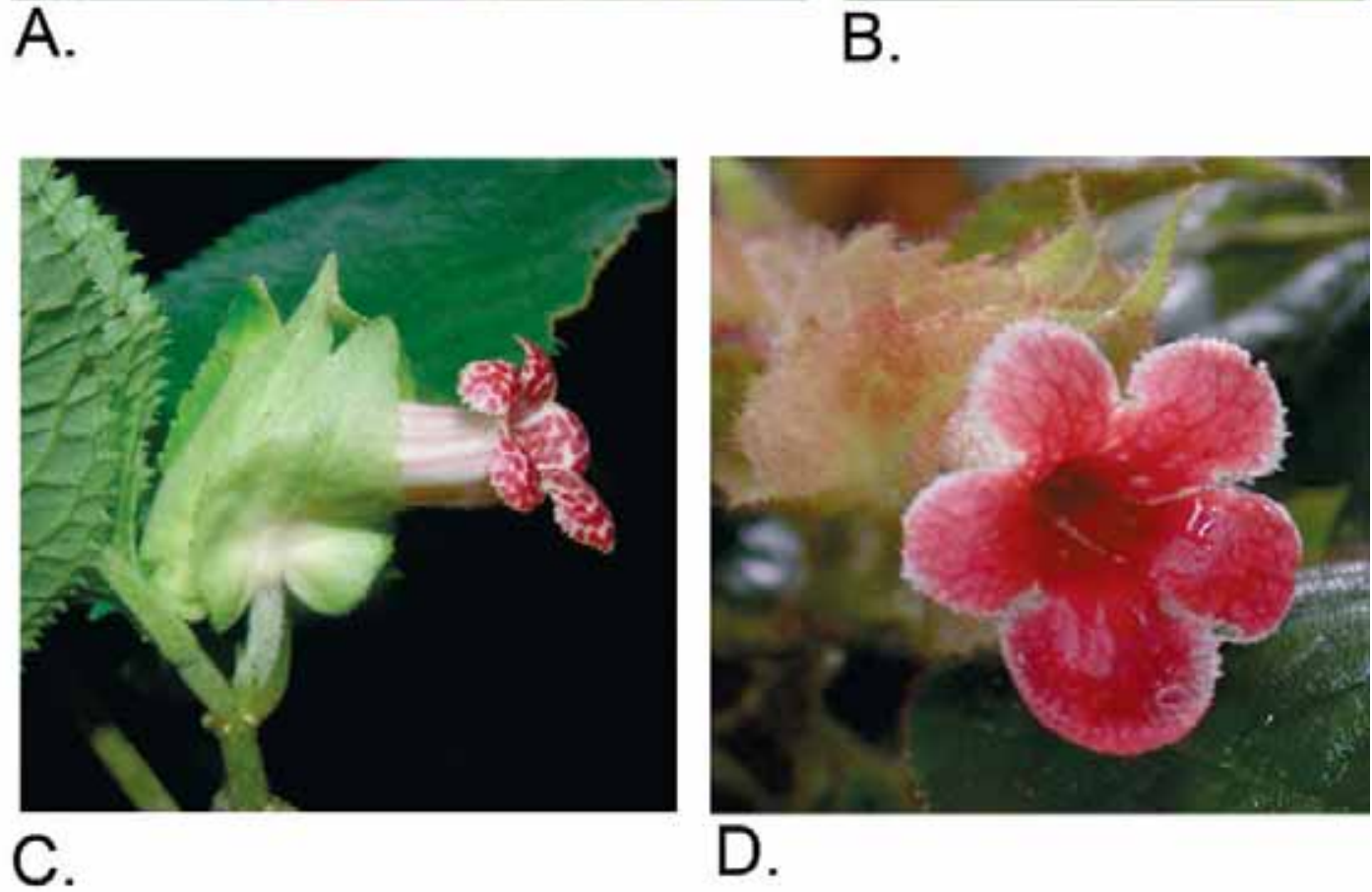

Fig. 1. Drymonia tomentulifera Kriebel. A. Flor en fase femenina. a. bráctea, b. pedicelo, c. lóbulo del cáliz, d. corola, e. filamentos, f. glándula nectarífera, g. ovario, h. estilo, i. estigma. B. Vista frontal. C. Vista lateral. D. Drymonia pilifera Wiehler. Fotos de D. tomentulifera son del paratipo R. Kriebel 5111 (INB). 